\title{
A NEW MATRIX INEQUALITY INVOLVING PARTIAL TRACES
}

\author{
Yongtao Li, Weijun LiU AND YANG HUANG*
}

Abstract. Let $A$ be an $m \times m$ positive semidefinite block matrix with each block being $n$-square. We write $\operatorname{tr}_{1}$ and $\operatorname{tr}_{2}$ for the first and second partial trace, respectively. In this paper, we prove the following inequality

$$
(\operatorname{tr} A) I_{m n}-\left(\operatorname{tr}_{2} A\right) \otimes I_{n} \geqslant \pm\left(I_{m} \otimes\left(\operatorname{tr}_{1} A\right)-A\right) .
$$

This inequality is not only a generalization of Ando's result [ILAS Conference (2014)] and Lin [Canad. Math. Bull. 59 (2016) 585-591], but it also could be regarded as a complement of a recent result of Choi [Linear Multilinear Algebra 66 (2018) 1619-1625]. Additionally, some new partial traces inequalities for positive semidefinite block matrices are also included.

Mathematics subject classification (2020): 15A45, 15A60, 47B65.

Keywords and phrases: Partial traces, block matrices, positive semidefinite, Cauchy-Khinchin.

\section{REFERENCES}

[1] T. ANDO, Matrix inequalities involving partial traces, ILAS Conference, 2014.

[2] T. Ando, Geometric mean and norm Schwarz inequality, Ann. Funct. Anal. 7 (2016) 1-8.

[3] R. Bhatia, Matrix Analysis, GTM 169, Springer-Verlag, New York, 1997.

[4] R. Bhatia, Positive Definite Matrices, Princeton University Press, Princeton, 2007.

[5] M. D. Chol, Positive linear maps on $C^{*}$-algebras, Canad. J. Math. 24 (1972) 520-529.

[6] D. CHOI, Inequalities related to partial transpose and partial trace, Linear Algebra Appl. 516 (2017) $1-7$.

[7] D. CHOI, Inequalities related to trace and determinant of positive semidefinite block matrices, Linear Algebra Appl. 532 (2017) 1-7.

[8] D. CHOI, Inequalities about partial transpose and partial trace, Linear Multilinear Algebra 66 (2018) $1619-1625$.

[9] E. R. van DaM, A Cauchy-Khinchin matrix inequality, Linear Algebra Appl. 280 (1998) 163-172.

[10] X. FU, P.-S. LAU, T.-Y. TAM, Linear maps of positive partial transpose matrices and singular value inequalities, Math. Inequal. Appl. 23 (4) (2020) 1459-1468.

[11] M. Gunus, J. Liu, S. RaOuAFI, T.-Y. TAm, Positive semi-definite $2 \times 2$ block matrices and norm inequalities, Linear Algebra Appl. 551 (2018) 83-91.

[12] R. A. Horn, C. R. Johnson, Matrix Analysis, 2nd ed., Cambridge University Press, Cambridge, 2013.

[13] F. Kittaneh, M. Lin, Trace inequalities for positive semidefinite block matrices, Linear Algebra Appl. 524 (2017) 153-158.

[14] E.-Y. LEE, The off-diagonal block of a PPT matrix, Linear Algebra Appl. 486 (2015) 449-453.

[15] Y. Li, Y. HUANG, L. Feng, W. LiU, Some applications of two completely copositive maps, Linear Algebra Appl. 590 (2020) 124-132.

[16] M. Lin, A completely PPT map, Linear Algebra Appl. 459 (2014) 404-410.

[17] M. LIN, Inequalities related to $2 \times 2$ block PPT matrices, Oper. Matrices, 9 (2015) 917-924.

[18] M. Lin, A determinantal inequality involving partial traces, Canad. Math. Bull. 59 (2016) 585-591.

[19] D. PetZ, Quantum Information Theory and Quantum Statistics. Theoretical and Mathematical Physics, Springer, Berlin, 2008. 
[20] P. Zhang, On some inequalities related to positive block matrices, Linear Algebra Appl. 576 (2019) 258-267.

[21] X. ZhaN, Matrix Inequalities, Springer, New York, 2002.

[22] X. Zhan, Matrix Theory, Graduate Studies in Mathematics, vol. 147, Amer. Math. Soc., Providence, RI, 2013.

[23] F. Zhang, Matrix Theory: Basic Results and Techniques, 2nd edition, Springer, New York, 2011.

[24] F. ZHANG, Positivity of matrices with generalized matrix functions, Acta Math. Sin. (Engl. Ser.) 28 (2012) 1779-1786. 